\title{
AN ALGORITHM TO COMPUTE THE CANONICAL BASIS OF AN IRREDUCIBLE MODULE OVER A QUANTIZED ENVELOPING ALGEBRA
}

\author{
WILLEM A. DE GRAAF
}

\begin{abstract}
The paper describes an algorithm to compute the canonical basis of an irreducible module over a quantized enveloping algebra of a finite-dimensional semisimple Lie algebra. The algorithm works for any module that is constructed as a submodule of a tensor product of modules with known canonical bases.
\end{abstract}

\section{Introduction}

In this paper we consider the problem of constructing the canonical basis (see [17]) of an irreducible module over a quantized enveloping algebra. There are several possible ways to approach this problem, and they may depend on how the module is constructed. In [4] an algorithm is described that works for any module, provided that we have a method for computing the action of elements of the algebra. In [11] and [12] the irreducible module is first constructed as a submodule of a tensor product of other modules. Then, using the known canonical bases of these other modules, an algorithm is described for constructing the canonical basis of the submodule.

Since constructing irreducible modules as submodules of tensor products can be quite efficient (see [5]), it would be worthwhile to have an algorithm tailored to this situation. Therefore, in this paper we take the second approach above. In fact, we describe an algorithm that is very similar to those in $[\mathbf{1 1}, \mathbf{1 2}]$. The main difference is that we do not assume that the root system is of a certain type. The algorithm given here works for all types, assuming that somehow we know the canonical bases of the fundamental modules. These can, for instance, be constructed using the algorithm of [4].

This paper is organised as follows. In Section 2 the theoretical concepts and the notation used in the paper are introduced. Then in Section 3 a result is described concerning the form of the elements of the canonical basis of a tensor product. In Section 4 this is used, along with the description of a monomial basis of an irreducible module (from [10]), to give an algorithm for constructing the canonical basis. Next, in Section 5 this algorithm is compared to the algorithm from [11] in the $A_{n}$-case. It is shown that in this case the two algorithms are very similar (but not exactly the same). In the final section, some examples of practical experiences with the algorithm are reported.

\section{Preliminaries}

In this section we briefly sketch the concepts and notation that we will be using. Our main reference is [6]. 
Let $\mathfrak{g}$ be a semisimple Lie algebra over $\mathbb{C}$. By $\Phi$ we denote the root system of $\mathfrak{g}$, and $\Delta=\left\{\alpha_{1}, \ldots, \alpha_{l}\right\}$ will denote a fixed set of simple roots of $\Phi$. Let $W$ denote the Weyl group of $\Phi$, which is generated by the simple reflections $s_{i}=s_{\alpha_{i}}$ for $1 \leqslant i \leqslant l$. Let $\mathbb{R} \Phi$ be the vector space over $\mathbb{R}$ spanned by $\Phi$. On $\mathbb{R} \Phi$ we fix a $W$-invariant inner product ( , ) such that $(\alpha, \alpha)=2$ for short roots $\alpha$. This means that $(\alpha, \alpha)=2,4,6$ for $\alpha \in \Phi$.

We work over the field $\mathbb{Q}(q)$. For $\alpha \in \Phi$, we set $q_{\alpha}=q^{(\alpha, \alpha) / 2}$. For $n \in \mathbb{Z}$, we set $[n]_{\alpha}=q_{\alpha}^{-n+1}+q_{\alpha}^{-n+3}+\ldots+q_{\alpha}^{n-1}$. Also $[n]_{\alpha} !=[n]_{\alpha}[n-1]_{\alpha} \ldots[1]_{\alpha}$ and

$$
\left[\begin{array}{l}
n \\
k
\end{array}\right]_{\alpha}=\frac{[n]_{\alpha} !}{[k]_{\alpha} ![n-k]_{\alpha} !}
$$

Let $\Delta=\left\{\alpha_{1}, \ldots, \alpha_{l}\right\}$ be a simple system of $\Phi$. Then the quantized enveloping algebra $U_{q}=U_{q}(\mathfrak{g})$ is the associative algebra (with an identity element) over $\mathbb{Q}(q)$ generated by $F_{\alpha}, K_{\alpha}, K_{\alpha}^{-1}$ and $E_{\alpha}$ for $\alpha \in \Delta$, subject to the following relations:

$$
\begin{gathered}
K_{\alpha} K_{\alpha}^{-1}=K_{\alpha}^{-1} K_{\alpha}=1, \\
K_{\alpha} K_{\beta}=K_{\beta} K_{\alpha}, \\
E_{\beta} K_{\alpha}=q^{-(\alpha, \beta)} K_{\alpha} E_{\beta}, \\
K_{\alpha} F_{\beta}=q^{-(\alpha, \beta)} F_{\beta} K_{\alpha}, \\
E_{\alpha} F_{\beta}=F_{\beta} E_{\alpha}+\delta_{\alpha, \beta} \frac{K_{\alpha}-K_{\alpha}^{-1}}{q_{\alpha}-q_{\alpha}^{-1}}, \\
\sum_{k=0}^{1-\left\langle\beta, \alpha^{\vee}\right\rangle}(-1)^{k}\left[\begin{array}{c}
1-\left\langle\beta, \alpha^{\vee}\right\rangle \\
k
\end{array}\right]_{\alpha} E_{\alpha}^{1-\left\langle\beta, \alpha^{\vee}\right\rangle-k} E_{\beta} E_{\alpha}^{k}=0, \\
\sum_{k=0}^{1-\left\langle\beta, \alpha^{\vee}\right\rangle}(-1)^{k}\left[\begin{array}{c}
1-\left\langle\beta, \alpha^{\vee}\right\rangle \\
k
\end{array}\right]_{\alpha} F_{\alpha}^{1-\left\langle\beta, \alpha^{\vee}\right\rangle-k} F_{\beta} F_{\alpha}^{k}=0,
\end{gathered}
$$

where the last two relations hold for all $\alpha \neq \beta$.

Let $U^{-}, U^{0}$ and $U^{+}$be the subalgebras of $U_{q}$ generated, respectively, by $F_{\alpha}$ for $\alpha \in \Delta$, $K_{\alpha}^{ \pm 1}$ for $\alpha \in \Delta$, and $E_{\alpha}$ for $\alpha \in \Delta$. Then, as a vector space, $U_{q} \cong U^{-} \otimes U^{0} \otimes U^{+}$(see [6, Theorem 4.21]). Let $v=\sum_{k} a_{k} \alpha_{k}$ with $a_{k} \in \mathbb{Z}_{\geqslant 0}$. Then we let $U_{v}^{+}$be the subspace of $U^{+}$spanned by all $E_{\alpha_{i_{1}}} \ldots E_{\alpha_{i_{r}}}$ such that $\alpha_{i_{1}}+\ldots+\alpha_{i_{r}}=v$. Similarly, $U_{v}^{-}$denotes the subspace of $U^{-}$spanned by all $F_{\alpha_{i_{1}}} \ldots F_{\alpha_{i_{r}}}$ such that $\alpha_{i_{1}}+\ldots+\alpha_{i_{r}}=v$.

We denote by $\lambda_{1}, \ldots, \lambda_{l}$ the fundamental weights, and by $P=\mathbb{Z} \lambda_{1}+\ldots+\mathbb{Z} \lambda_{l}$, the weight lattice. Also, $P^{+}=\mathbb{Z}_{\geqslant 0} \lambda_{1}+\ldots+\mathbb{Z}_{\geqslant 0} \lambda_{l}$ is the set of dominant weights. Now, for every dominant $\lambda \in P^{+}$, there is an irreducible $U_{q}$-module $V(\lambda)$. The module $V(\lambda)$ is spanned by vectors $v_{\mu}$ for $\mu \in P$, with $K_{\alpha} \cdot v_{\mu}=q^{(\mu, \alpha)} v_{\mu}$. These $v_{\mu}$ are called weight-vectors of weight $\mu$. Among them there is the vector $v_{\lambda}$ (which is unique up to scalar multiples), with $U^{+} \cdot v_{\lambda}=0$. This $v_{\lambda}$ is called the highest-weight vector. We find that $V(\lambda)=U^{-} \cdot v_{\lambda}$. Furthermore, every finite-dimensional irreducible $U_{q}$-module is isomorphic to a $V(\lambda)$; see [6, Theorem 5.10].

Let $M$ be a finite-dimensional $U_{q}$-module. Then $M$ has a crystal base $(\mathcal{M}, \mathscr{B})$ as defined in $[6,9.4]$. Here $\mathcal{M}$ is an $A$-submodule of $M$, where $A$ is the subring of $\mathbb{Q}(q)$ consisting of rational functions without pole at 0 , and $\mathcal{B}$ is a basis of $\mathcal{M} / q \mathcal{M}$. For $\alpha \in \Delta$, we have the Kashiwara operators $\widetilde{F}_{\alpha}, \widetilde{E}_{\alpha}: \mathcal{M} \rightarrow \mathcal{M}$, and the induced operators $\widetilde{F}_{\alpha}, \widetilde{E}_{\alpha}: \mathcal{B} \rightarrow \mathcal{B} \cup\{0\}$; see $[6,9.2,9.4]$. 
There is a $\mathbb{Q}$-algebra isomorphism

$$
\text { - }: U_{q} \rightarrow U_{q}, \quad \text { with } \bar{q}=q^{-1}, \bar{E}_{\alpha}=E_{\alpha}, \bar{F}_{\alpha}=F_{\alpha} \text { and } \bar{K}_{\alpha}=K_{\alpha}^{-1} \text {; }
$$

see [6, Proposition 11.9]. If $V(\lambda)$ is an irreducible $U_{q}$-module with highest weight $\lambda$, and fixed highest-weight vector $v_{\lambda}$, then we have an induced map

$$
-: V(\lambda) \rightarrow V(\lambda) \text { by } \overline{u \cdot v_{\lambda}}=\bar{u} \cdot v_{\lambda} .
$$

(This is well defined by [6, Proposition 11.9].) The fixed choice for $v_{\lambda}$ leads to a fixed crystal base $(\mathcal{L}(\lambda), \mathcal{B}(\lambda))$ of $V(\lambda)$, where $\mathcal{L}(\lambda)$ is spanned by all $\widetilde{F}_{\alpha_{i_{1}}} \ldots \widetilde{F}_{\alpha_{i_{r}}}\left(v_{\lambda}\right)$, for $r \geqslant 0$. Now (for example, by [16, Theorem 1.8] and [6, Theorem 11.10]) there is a unique basis $\left\{G_{\lambda}(b) \mid b \in \mathcal{B}(\lambda)\right\}$ of $\mathcal{L}(\lambda)$, such that:

1. $G_{\lambda}(b)=b \bmod q \mathcal{L}(\lambda)$;

2. $\overline{G_{\lambda}(b)}=G_{\lambda}(b)$.

This basis is called the canonical basis of $V(\lambda)$.

In the discussion that follows, when we write 'the crystal base' or 'the canonical basis' of $V(\lambda)$, we are always assuming that a fixed highest-weight vector $v_{\lambda}$ has been chosen, which makes the choice of the crystal base or the canonical basis unique.

The crystal graph $\Gamma_{\lambda}$ of the module $V(\lambda)$ is defined as follows. The points of $\Gamma_{\lambda}$ are the elements of $\mathcal{B}(\lambda)$, and there is an edge $b_{1} \stackrel{\alpha}{\longrightarrow} b_{2}$ if $\widetilde{F}_{\alpha}\left(b_{1}\right)=b_{2}$. There is a very elegant method of computing the crystal graph, using Littelmann's path method. Let $\mathbb{R} P$ be the vector space over $\mathbb{R}$ spanned by the weights. Let $\Pi$ be the set of piecewise linear paths $\pi:[0,1] \rightarrow \mathbb{R} P$, such that $\pi(0)=0$. For $\alpha \in \Delta$, Littelmann defined operators $e_{\alpha}, f_{\alpha}: \Pi \rightarrow \Pi \cup\{0\}$ (see $[13,14]$ ), with the following property. Let $\lambda \in P^{+}$be a dominant weight, and let $\pi_{\lambda}$ be the path given by $\pi_{\lambda}(t)=\lambda t$ (that is, a straight line from the origin to $\lambda)$. Let $\Pi_{\lambda}$ be the set of all $f_{\alpha_{i_{1}}} \ldots f_{\alpha_{i_{k}}}\left(\pi_{\lambda}\right)$. Then all paths in $\Pi_{\lambda}$ end in an element of $P$. Furthermore, the number of paths ending in $\mu \in P$ is equal to the dimension of the weight space with weight $\mu$ in the irreducible $U_{q}$-module $V(\lambda)$.

Now we consider the directed labeled graph with point set $\Pi_{\lambda}$, and edges $\pi_{1} \stackrel{\alpha}{\longrightarrow} \pi_{2}$ if $f_{\alpha}\left(\pi_{1}\right)=\pi_{2}$. This graph is isomorphic to the crystal graph of $V(\lambda)$; see [8].

Let $M_{1}$ and $M_{2}$ be $U_{q}$-modules; then $M_{1} \otimes M_{2}$ is a $U_{q}$-module via the comultiplication of $U_{q}$. There are many possible ways of defining this, and the comultiplication $\Delta: U_{q} \rightarrow$ $U_{q} \otimes U_{q}$ that we use is given by

$$
\begin{aligned}
& \Delta\left(E_{\alpha}\right)=E_{\alpha} \otimes K_{\alpha}^{-1}+1 \otimes E_{\alpha} \\
& \Delta\left(F_{\alpha}\right)=F_{\alpha} \otimes 1+K_{\alpha} \otimes F_{\alpha} \\
& \Delta\left(K_{\alpha}\right)=K_{\alpha} \otimes K_{\alpha}
\end{aligned}
$$

see $[6,9.13]$.

\section{Canonical bases of tensor products}

Here we give a description of the canonical basis of a tensor product, following [6, Chapter 9] and [17, 27.3].

Let $V(\mu)$ and $V\left(\mu^{\prime}\right)$ be two irreducible $U_{q}$-modules, with highest weights $\mu$ and $\mu^{\prime}$. Let $C=\left\{v_{1}, \ldots, v_{m}\right\}$ and $C^{\prime}=\left\{v_{1}^{\prime}, \ldots, v_{n}^{\prime}\right\}$ be fixed canonical bases of $V(\mu)$ and $V\left(\mu^{\prime}\right)$. Denote the weights of $v_{i}$ and $v_{j}^{\prime}$ by $v_{i}$ and $v_{j}^{\prime}$, respectively. Then $v_{i}=\mu-\sum_{k} a_{k, i} \alpha_{k}$ with $a_{k, i} \in \mathbb{Z}_{\geqslant 0}$, and we say that $\sum_{k} a_{k, i}$ is the height of $v_{i}$. The height of $v_{j}^{\prime}$ is defined similarly. 
We assume that the bases $C$ and $C^{\prime}$ are ordered according to increasing height. So $v_{1}=v_{\mu}$ and $v_{1}^{\prime}=v_{\mu^{\prime}}$ are the highest-weight vectors.

Let $(\mathcal{L}, \mathscr{B})$ and $\left(\mathcal{L}^{\prime}, \mathcal{B}^{\prime}\right)$ be crystal bases of $V(\mu)$ and $V\left(\mu^{\prime}\right)$ respectively. Here $\mathcal{L}$ and $\mathcal{L}^{\prime}$ are spanned by $C$ and $C^{\prime}$, respectively. Furthermore, $\mathcal{B}$ and $\mathcal{B}^{\prime}$ consist of the cosets $v_{i} \bmod q \mathcal{L}$ and $v_{j}^{\prime} \bmod q \mathcal{L}^{\prime}$. Now, by [6, Theorem 9.17], $\left(\mathcal{L} \otimes \mathcal{L}^{\prime}, \mathscr{B} \otimes \mathscr{B}^{\prime}\right)$ is a crystal base of $V(\mu) \otimes V\left(\mu^{\prime}\right)$.

We let $\Theta$ be the element from $[\mathbf{1 7}, 4.1]$, and then

$$
P: U_{q} \otimes U_{q} \rightarrow U_{q} \otimes U_{q}
$$

is the algebra homomorphism defined by $P(a \otimes b)=b \otimes a$. We set $\Theta^{0}=P(\bar{\Theta})$; then $\Theta^{0}=\sum_{\eta \geqslant 0} \Theta_{\eta}^{0}$, where the sum runs over all $\eta=\sum_{k} b_{k} \alpha_{k}$ with $b_{k} \in \mathbb{Z}_{\geqslant 0}$. Furthermore,

$$
\Theta_{\eta}^{0} \in U_{\eta}^{+} \otimes U_{\eta}^{-} \quad \text { and } \quad \Theta_{0}^{0}=1 \otimes 1 .
$$

Now $\Psi_{0}: V(\mu) \otimes V\left(\mu^{\prime}\right) \rightarrow V(\mu) \otimes V\left(\mu^{\prime}\right)$ is the map defined by $\Psi_{0}\left(v \otimes v^{\prime}\right)=\Theta^{0}\left(\bar{v} \otimes \bar{v}^{\prime}\right)$.

Lemma I. We have $\Psi_{0}\left(u \cdot v \otimes v^{\prime}\right)=\bar{u} \cdot \Psi_{0}\left(v \otimes v^{\prime}\right)$ for all $u \in U^{-}$. Furthermore, $\Psi_{0}^{2}\left(v \otimes v^{\prime}\right)=$ $v \otimes v^{\prime}$ for all $v \in V(\mu), v^{\prime} \in V\left(\mu^{\prime}\right)$.

Proof. This is the same as the proof of the corresponding results in [17, 27.3.1]. The difference is that we use a different comultiplication. Denoting the comultiplication used in [17] by $\Delta_{L}$, we have $\Delta_{L}\left(F_{\alpha}\right)=F_{\alpha} \otimes K_{\alpha}^{-1}+1 \otimes F_{\alpha}$. This means that for $u \in U^{-}$, we have

$$
\Delta(u)=P\left(\bar{\Delta}_{L}(u)\right), \quad \text { where } \quad \bar{\Delta}_{L}(u)=\overline{\Delta_{L}(\bar{u})} .
$$

The property that $\Delta_{L}(u) \Theta=\Theta \bar{\Delta}_{L}(u)$ (see [17, Theorem 4.1.2]) now translates to

$$
\Delta(u) \Theta^{0}=\Theta^{0} \bar{\Delta}(u),
$$

where $\bar{\Delta}$ is defined similarly to $\bar{\Delta}_{L}$. From this, the first statement follows. The second follows from $\Theta^{0} \bar{\Theta}^{0}=1 \otimes 1$; see [17, Corollary 4.1.3].

We define a partial order on the $v_{i} \otimes v_{j}^{\prime}$. We set $v_{i} \otimes v_{j}^{\prime}<v_{k} \otimes v_{l}^{\prime}$ if and only if $i<k$, $j>l$, and $v_{i}+v_{j}^{\prime}=v_{k}+v_{l}^{\prime}$.

Proposition 2. There are unique elements $w_{i j} \in V(\mu) \otimes V\left(\mu^{\prime}\right)$ such that

(i) $\Psi_{0}\left(w_{i j}\right)=w_{i j}$;

(ii) $w_{i j}=v_{i} \otimes v_{j}^{\prime}+\sum_{k} \zeta_{k} v_{i_{k}} \otimes v_{j_{k}}^{\prime}$, with $\zeta_{k} \in q \mathbb{Z}[q]$, and $v_{i_{k}}+v_{j_{k}}^{\prime}=v_{i}+v_{j}^{\prime}$.

Also, $v_{i_{k}} \otimes v_{j_{k}}^{\prime}<v_{i} \otimes v_{j}^{\prime}$ for all $k$. The elements $w_{i j}$ form a basis of $V(\mu) \otimes V\left(\mu^{\prime}\right)$.

Proof. This proceeds in the same way as the proof of [17, Theorem 27.3.2]. Note that

$$
\Psi_{0}\left(v_{i} \otimes v_{j}\right)=v_{i} \otimes v_{j}+\sum_{k} \xi_{k} v_{i_{k}} \otimes v_{j_{k}}^{\prime},
$$

with $\xi_{k} \in \mathbb{Z}\left[q, q^{-1}\right]$. From $\Theta_{\eta}^{0} \in U_{\eta}^{+} \otimes U_{\eta}^{-}$and the assumption on the ordering of $C$ and $C^{\prime}$, it follows that $v_{i_{k}} \otimes v_{j_{k}}^{\prime}<v_{i} \otimes v_{j}^{\prime}$ for all $k$. Let $X$ be the set of all $(i, j)$ with $v_{i}+v_{j}^{\prime}=v$, for a certain $v$. Order the elements of $X$ in such a way that $v_{i} \otimes v_{j}^{\prime}<v_{k} \otimes v_{l}^{\prime}$ implies that $(i, j)$ appears before $(k, l)$. Let $(i, j)$ be the smallest element of $X$. Then, by $(1)$, we see that $\Psi_{0}\left(v_{i} \otimes v_{j}^{\prime}\right)=v_{i} \otimes v_{j}^{\prime}$. So in this case we set $w_{i j}=v_{i} \otimes v_{j}^{\prime}$. Now choose a $(k, l) \in X$, and suppose that $w_{r, s}$ exist for all $(r, s) \in X$ appearing before $(k, l)$. Then, using (1) and 
the triangular form of the $w_{r, s}$, we can write $\Psi_{0}\left(v_{k} \otimes v_{l}^{\prime}\right)-v_{k} \otimes v_{l}^{\prime}=\sum_{r, s} \zeta_{r, s} w_{r, s}$, where $v_{r} \otimes v_{s}^{\prime}<v_{k} \otimes v_{l}^{\prime}$. After taking images under $\Psi_{0}$, and using the fact that $\Psi_{0}$ is an involution, we see that the $\zeta_{r, s} \in \mathbb{Z}\left[q, q^{-1}\right]$ satisfy $\bar{\zeta}_{r, s}=-\zeta_{r, s}$. This implies that there are unique $\delta_{r, s} \in q \mathbb{Z}[q]$ with $\zeta_{r, s}=\delta_{r, s}-\bar{\delta}_{r, s}$. Now set $w_{k, l}=v_{k} \otimes v_{l}^{\prime}+\sum_{r, s} \delta_{r, s} w_{r, s}$. For the uniqueness, suppose that there are $w_{i j}^{\prime} \in V(\mu) \otimes V\left(\mu^{\prime}\right)$ satisfying conditions (i) and (ii). Then we write $w_{i j}^{\prime}$ as a linear combination of $w_{i j}$. By condition (ii), the coefficients are in $\mathbb{Z}[q]$. Then condition (i) implies that they are in $\mathbb{Z}$. Finally, from condition (ii) we see that one coefficient is 1 , and the others are 0 .

Let $V(\lambda)$ denote the $U_{q}$-submodule of $V(\mu) \otimes V\left(\mu^{\prime}\right)$ generated by $v_{\mu} \otimes v_{\mu^{\prime}}=v_{1} \otimes v_{1}^{\prime}$. So $V(\lambda)$ is the irreducible $U_{q}$-module with highest weight $\lambda=\mu+\mu^{\prime}$. Set $\mathcal{L}(\lambda)=$ $\left(\mathscr{L} \otimes \mathcal{L}^{\prime}\right) \cap V(\lambda)$, and $\mathscr{B}(\lambda)=\left(\mathscr{B} \otimes \mathscr{B}^{\prime}\right) \cap \mathcal{L}(\lambda) / q \mathcal{L}(\lambda)$. Then, by [6, Proposition 9.10], $(\mathscr{L}(\lambda), \mathscr{B}(\lambda))$ is a crystal base of $V(\lambda)$ (the hypotheses of this proposition are satisfied by [6, Proposition 9.23 and Lemma 9.26]).

THEOREM 3. The elements of the canonical basis of $V(\lambda)$ have the form

$$
v_{i} \otimes v_{j}^{\prime}+\sum_{k} \zeta_{k} v_{i_{k}} \otimes v_{j_{k}}^{\prime},
$$

with $\zeta_{k} \in q \mathbb{Z}[q]$, and $v_{i_{k}} \otimes v_{j_{k}}^{\prime}<v_{i} \otimes v_{j}^{\prime}$ for all $k$.

Proof. We know that $\Psi_{0}\left(v_{1} \otimes v_{1}^{\prime}\right)=v_{1} \otimes v_{1}^{\prime}$.

So by Lemma $1, \Psi_{0}$ coincides with ${ }^{-}$on $V(\lambda)$ (where $\left.\overline{u \cdot v_{1} \otimes v_{1}^{\prime}}=\bar{u} \cdot v_{1} \otimes v_{1}^{\prime}\right)$. Hence the elements of the canonical basis of $V(\lambda)$ are invariant under $\Psi_{0}$. Also, since the elements of the canonical basis lie in $\mathcal{L}(\lambda)$ and are equal to a $v_{i} \otimes v_{j}^{\prime} \bmod q \mathcal{L}(\lambda)$, they must be of the form $v_{i} \otimes v_{j}+\sum_{k} \zeta_{k} v_{i_{k}} \otimes v_{j_{k}}^{\prime}$ with $\zeta_{k} \in q \mathbb{Z}[q]$. Now Proposition 2 finishes the proof.

Now let $V\left(\mu_{1}\right), \ldots, V\left(\mu_{r}\right)$ be irreducible $U_{q}$-modules with canonical bases $C_{i}$ $=\left\{v_{1}^{i}, \ldots, v_{m_{i}}^{i}\right\}$, ordered according to increasing height. We consider the tensor product $V=V\left(\mu_{1}\right) \otimes \ldots \otimes V\left(\mu_{r}\right)$. We write

$$
v_{i_{1}}^{1} \otimes \ldots \otimes v_{i_{r}}^{r}<_{\text {lex }} v_{j_{1}}^{1} \otimes \ldots \otimes v_{j_{r}}^{r}
$$

if there is a $k$ with $i_{1}=j_{1}, \ldots, i_{k}=j_{k}$ and $i_{k+1}<j_{k+1}$. Set $\lambda=\mu_{1}+\ldots+\mu_{r}$ and let $V(\lambda)$ be the $U_{q}$-submodule of $V$ generated by $v_{1}^{1} \otimes \ldots \otimes v_{1}^{r}$.

COROLlaRY 4. The elements of the canonical basis of $V(\lambda)$ have the form

$$
v_{i_{1}}^{1} \otimes \ldots \otimes v_{i_{r}}^{r}+\sum_{k} \zeta_{k} x_{k}
$$

where $\zeta_{k} \in q \mathbb{Z}[q], x_{k} \in C_{1} \otimes \ldots \otimes C_{r}$, and $x_{k}<\operatorname{lex} v_{i_{1}}^{1} \otimes \ldots \otimes v_{i_{r}}^{r}$.

Proof. The case $r=2$ is covered by Theorem 3, so suppose that $r>2$. Let $W$ be the $U_{q^{-}}$ submodule of $V\left(\mu_{2}\right) \otimes \ldots \otimes V\left(\mu_{r}\right)$ generated by $v_{1}^{2} \otimes \ldots \otimes v_{1}^{r}$. Then $W$ is the irreducible $U_{q}$-module with highest weight $\mu_{2}+\ldots+\mu_{r}$. Let $\left\{w_{1}, \ldots, w_{s}\right\}$ be the canonical basis of $W$. Then by Theorem 3 the elements of the canonical basis of $V(\lambda)$ have the form

$$
v_{i_{1}}^{1} \otimes w_{j_{1}}+\sum_{k \geqslant 2} \zeta_{k} v_{i_{k}}^{1} \otimes w_{j_{k}},
$$

with $i_{k}<i_{1}$ for all $k \geqslant 2$, and $\zeta_{k} \in q \mathbb{Z}[q]$. We obtain the result by writing all $w_{j_{k}}$ for $k \geqslant 1$ as linear combinations of elements of $C_{2} \otimes \ldots \otimes C_{r}$, and then using induction. 


\section{A monomial basis of $V(\lambda)$}

In this section we first describe a basis of $V(\lambda)$, following [10]. Then, using this, we derive an algorithm for constructing the canonical basis of $V(\lambda)$, when $V(\lambda)$ is viewed as a submodule of a tensor product.

Let $\pi \in \Pi_{\lambda}$. Then the first direction of $\pi$ is $w(\lambda)$ for some $w \in W / W_{\lambda}$ (see $[13,5.2]$ ), where $W_{\lambda}$ is the stabilizer of $\lambda$. Set $\phi(\pi)=w$. Let $s_{i_{1}} \ldots s_{i_{r}}$ be the reduced expression for $\phi(\pi)$, which is lexicographically the smallest. (Here, $s_{i_{1}} \ldots s_{i_{r}}$ is lexicographically smaller than $s_{j_{1}} \ldots s_{j_{r}}$ if there is a $k>0$ such that $i_{1}=j_{1}, \ldots, i_{k-1}=j_{k-1}$ and $i_{k}<j_{k}$.) Then we define integers $n_{1}, \ldots, n_{r}$, and paths $\pi_{0}, \pi_{1}, \ldots, \pi_{r}$ in the following way. First, $\pi_{0}=\pi$. We let $n_{k}$ be maximal such that $e_{\alpha_{i_{k}}}^{n_{k}} \pi_{k-1} \neq 0$, and we set $\pi_{k}=e_{\alpha_{i_{k}}}^{n_{k}} \pi_{k-1}$. Set

$$
\eta_{\pi}=\left(n_{1}, \ldots, n_{r}\right) \quad \text { and } \quad F_{\pi}=F_{\alpha_{i_{1}}}^{\left(n_{1}\right)} \ldots F_{\alpha_{i_{r}}}^{\left(n_{r}\right)} .
$$

Let $b_{\lambda} \in \mathscr{B}(\lambda)$ denote the unique element of weight $\lambda$ (it is the coset of $v_{\lambda}$ modulo $q \mathcal{L}(\lambda)$ ). Set

$$
b_{\pi}=\widetilde{F}_{\alpha_{i_{1}}}^{n_{1}} \ldots \widetilde{F}_{\alpha_{i_{r}}}^{n_{r}}\left(b_{\lambda}\right)
$$

then $\mathcal{B}(\lambda)=\left\{b_{\pi} \mid \pi \in \Pi_{\lambda}\right\}$ (this follows from [8]). In the discussion that follows, we let $<_{B}$ denote the Bruhat order on the Weyl group $W$. The lexicographical order on sequences of length $r$ is defined by $\left(m_{1}, \ldots, m_{r}\right)<_{\operatorname{lex}}\left(n_{1}, \ldots, n_{r}\right)$ if there is a $k$ such that $m_{1}=n_{1}, \ldots, m_{k-1}=n_{k-1}$ and $m_{k}<n_{k}$. We now define a partial order on $\Pi_{\lambda}$ as follows. First of all, $\pi<\sigma$ if $\phi(\pi)<_{B} \phi(\sigma)$. Secondly, if $\phi(\pi)=\phi(\sigma)$, then $\pi<\sigma$ if $\eta_{\pi}>_{\text {lex }} \eta_{\sigma}$. For the proof of the folowing theorem we refer to [10].

\section{THEOREM 5.}

where $\zeta_{\pi, \sigma} \in \mathbb{Z}\left[q, q^{-1}\right]$.

$$
F_{\pi} \cdot v_{\lambda}=G_{\lambda}\left(b_{\pi}\right)+\sum_{\sigma<\pi} \zeta_{\pi, \sigma} G_{\lambda}\left(b_{\sigma}\right)
$$

Corollary 6. The set $\left\{F_{\pi} \cdot v_{\lambda} \mid \pi \in \Pi_{\lambda}\right\}$ is a basis of $V(\lambda)$.

Let $\pi \in \Pi_{\lambda}$, and $F_{\pi}=F_{\alpha_{i_{1}}}^{\left(n_{1}\right)} \ldots F_{\alpha_{i_{r}}}^{\left(n_{r}\right)}$. Then we say that $\pi$ is of weight $\nu=\sum_{k} n_{k} \alpha_{i_{k}}$. We note that this means that $F_{\pi} \cdot v_{\lambda}$ is a weight vector in $V(\lambda)$ of weight $\lambda-v$. By $\Pi_{\lambda, v}$, we denote the set of all $\pi \in \Pi_{\lambda}$ of weight $\nu$.

Suppose that $\lambda=\mu_{1}+\ldots+\mu_{r}$, where the $\mu_{i}$ are dominant weights. Also suppose that we are given the modules $V\left(\mu_{i}\right)$ with canonical bases $C_{i}=\left\{v_{1}^{i}, \ldots, v_{m_{i}}^{i}\right\}$, ordered according to increasing height. We identify $V(\lambda)$ with the $U_{q}$-submodule of $V\left(\mu_{1}\right) \otimes \ldots \otimes$ $V\left(\mu_{r}\right)$ generated by $v_{\lambda}=v_{1}^{1} \otimes \ldots \otimes v_{r}^{1}$. Set $C=C_{1} \otimes \ldots \otimes C_{r}$, which is a basis of $V\left(\mu_{1}\right) \otimes \ldots \otimes V\left(\mu_{r}\right)$, ordered with respect to $<_{\text {lex }}$ (see the previous section).

Theorem 5 leads to the following algorithm for computing the $G_{\lambda}\left(b_{\pi}\right)$, for $\pi \in \Pi_{\lambda, \nu}$. Let $\sigma_{1}, \ldots, \sigma_{r}$ be the elements from $\Pi_{\lambda, v}$ that are smaller than $\pi$. We assume that the $G_{\lambda}\left(b_{\sigma_{i}}\right)$ have already been computed. Write $G_{\lambda}\left(b_{\sigma_{i}}\right)=y_{i}+\sum_{k} \zeta_{k} y_{i, k}$, where $y_{i}, y_{i, k} \in C$ and $y_{i}>_{\text {lex }} y_{i, k}$ for all $k$. We assume that $y_{i}<_{\operatorname{lex}} y_{j}$ implies that $i>j$. Then we proceed as follows.

1. Write $X=F_{\pi} \cdot v_{\lambda}$ as a linear combination of elements of $C$.

2. For $i=1, \ldots, r$, we act as follows.

Let $\zeta_{i}$ be the coefficient of $y_{i}$ in $X$. Let $\xi_{i}$ be the unique element of $\mathbb{Z}\left[q, q^{-1}\right]$ such that $\bar{\xi}_{i}=\xi_{i}$ and $\zeta_{i}+\xi_{i} \in q \mathbb{Z}[q]$.

Set $X:=X+\xi_{i} G_{\lambda}\left(b_{\sigma_{i}}\right)$. 
Proposition 7. When the loop in Step 2 terminates, we have $X=G_{\lambda}\left(b_{\pi}\right)$.

Proof. Note that by Theorem 5 there are coefficients $\xi_{i}$ such that

$$
G_{\lambda}\left(b_{\pi}\right)=F_{\pi} \cdot v_{\lambda}+\sum_{i=1}^{r} \xi_{i} G_{\lambda}\left(b_{\sigma_{i}}\right) .
$$

This implies that $\bar{\xi}_{i}=\xi_{i}$. Also, by Corollary 4, we know that $G_{\lambda}\left(b_{\pi}\right)$ is of the form $x+\sum_{k} \omega_{k} x_{k}$, where $x, x_{k} \in C$ and $\omega_{k} \in q \mathbb{Z}[q]$. Note that by Corollary 4, $y_{1}$ does not occur in any $G_{\lambda}\left(b_{\sigma_{i}}\right)$, except for $G_{\lambda}\left(b_{\sigma_{1}}\right)$. Therefore, $\xi_{1}$ is uniquely determined by the requirements that it should be invariant under ${ }^{-}$, and $\zeta_{1}+\xi_{i} \in q \mathbb{Z}[q]$. Then, in the same way, we see that $\xi_{2}$ is uniquely determined, and so on.

ExAmple 8 . Let $\Phi$ be the root system of type $G_{2}$. We denote the simple roots of $\Phi$ by $\alpha$ and $\beta$, where $\beta$ is long. The fundamental module $V\left(\lambda_{1}\right)$ is seven-dimensional, and the canonical basis is $C_{1}=\left\{v_{1}, \ldots, v_{7}\right\}$; these are weight vectors of weights $(1,0),(-1,1)$, $(2,-1),(0,0),(-2,1),(1,-1)$ and $(-1,0)$. Here we abbreviate a weight $m \lambda_{1}+n \lambda_{2}$ as $(m, n)$. The fundamental module $V\left(\lambda_{2}\right)$ is fourteen-dimensional and has canonical basis $C_{2}=\left\{w_{1}, \ldots, w_{14}\right\}$. The $w_{i}$ are weight vectors of weights $(0,1),(3,-1),(1,0),(-1,1)$, $(-3,2),(2,-1),(0,0),(0,0),(3,-2),(-2,1),(1,-1),(-1,0),(-3,1)$ and $(0,-1)$. A description of the action of the generators of $U_{q}$ on $V\left(\lambda_{1}\right)$ can, for instance, be found in [7], and the action of $U_{q}$ on $V\left(\lambda_{2}\right)$ is described in [6,5A.4]. Alternatively, these modules can be constructed using the GAP4 package QuaGroup [2, 3]. This package has been used to perform many of the calculations used in the rest of this example. Now we set $\lambda=2 \lambda_{1}+\lambda_{2}$. Then $V(\lambda)$ is the submodule of $W=V\left(\lambda_{1}\right) \otimes V\left(\lambda_{1}\right) \otimes V\left(\lambda_{2}\right)$ generated by $v_{1} \otimes v_{1} \otimes w_{1}$. We construct the elements of the canonical basis of $V(\lambda)$ that are of weight $\mu=(-2,2)$. We use the following elements of weight $\mu$ :

$$
\begin{aligned}
& x_{1}=v_{1} \otimes v_{2} \otimes w_{10} ; \quad x_{2}=v_{1} \otimes v_{4} \otimes w_{5} ; \quad x_{3}=v_{1} \otimes v_{5} \otimes w_{4} ; \\
& x_{4}=v_{2} \otimes v_{1} \otimes w_{10} ; \quad x_{5}=v_{2} \otimes v_{2} \otimes w_{7} ; \quad x_{6}=v_{2} \otimes v_{2} \otimes w_{8} ; \\
& x_{7}=v_{2} \otimes v_{3} \otimes w_{5} ; \quad x_{8}=v_{2} \otimes v_{4} \otimes w_{4} ; \quad x_{9}=v_{2} \otimes v_{5} \otimes w_{3} ; \\
& x_{10}=v_{2} \otimes v_{7} \otimes w_{1} ; \quad x_{11}=v_{3} \otimes v_{2} \otimes w_{5} ; \quad x_{12}=v_{4} \otimes v_{1} \otimes w_{5} ; \\
& x_{13}=v_{4} \otimes v_{2} \otimes w_{4} ; \quad x_{14}=v_{4} \otimes v_{5} \otimes w_{1} ; \quad x_{15}=v_{5} \otimes v_{1} \otimes w_{4} ; \\
& x_{16}=v_{5} \otimes v_{2} \otimes w_{3} ; \quad x_{17}=v_{5} \otimes v_{4} \otimes w_{1} ; \quad x_{18}=v_{7} \otimes v_{2} \otimes w_{1} .
\end{aligned}
$$

They are listed in lexicographical order; that is, $x_{1}<_{\operatorname{lex}} x_{2}<_{\operatorname{lex}} \ldots<_{\operatorname{lex}} x_{18}$. The weight space of weight $\mu$ in $V(\lambda)$ is five-dimensional. So we have five paths $\pi_{i}$ in the crystal graph. The corresponding words in the Weyl group are

$$
\begin{aligned}
& \phi\left(\pi_{1}\right)=s_{\alpha} s_{\beta} s_{\alpha} \\
& \phi\left(\pi_{2}\right)=s_{\beta} s_{\alpha} s_{\beta} \\
& \phi\left(\pi_{3}\right)=s_{\alpha} s_{\beta} s_{\alpha} \\
& \phi\left(\pi_{4}\right)=s_{\alpha} s_{\beta} s_{\alpha} s_{\beta} \\
& \phi\left(\pi_{5}\right)=s_{\alpha} s_{\beta} s_{\alpha} s_{\beta} s_{\alpha} .
\end{aligned}
$$

Setting $\eta_{i}=\eta_{\pi_{i}}$ we have $\eta_{1}=(4,2,1), \eta_{2}=(1,5,1), \eta_{3}=(3,2,2), \eta_{4}=(3,1,2,1)$ and $\eta_{5}=(2,1,2,1,1)$. So we see that $\pi_{1}<\pi_{3}<\pi_{4}<\pi_{5}$ and $\pi_{2}<\pi_{4}$. 


\section{Computing canonical bases}

Therefore we have

$$
\begin{aligned}
G_{\lambda}\left(b_{\pi_{1}}\right) & =F_{\pi_{1}} v_{\lambda} \\
& =x_{16}+q^{2} x_{15}+q^{3} x_{13}+q^{6} x_{12}+q^{8} x_{11}+q x_{9}+q^{3} x_{8}+q^{7} x_{7}+q^{5} x_{3}+q^{8} x_{2} ; \\
G_{\lambda}\left(b_{\pi_{2}}\right) & =F_{\pi_{2}} v_{\lambda}=x_{11}+q^{3} x_{7}+q^{6} x_{6} .
\end{aligned}
$$

Also,

$$
F_{\pi_{3}} v_{\lambda}=x_{17}+q^{2} x_{16}+q^{2} x_{14}+q^{3} x_{13}+q^{6} x_{11}+q^{3} x_{9}+q^{5} x_{8}+q^{9} x_{7} .
$$

All the coefficients, except the first one, are in $q \mathbb{Z}[q]$. Hence $G_{\lambda}\left(b_{\pi_{3}}\right)=F_{\pi_{3}} v_{\lambda}$. Now

$$
\begin{aligned}
F_{\pi_{4}} v_{\lambda}= & \left(q+q^{-1}\right) x_{16}+\left(q+q^{3}\right) x_{15}+\left(1+q^{2}+q^{4}\right) x_{13}+\left(q^{3}+q^{5}+q^{7}\right) x_{12} \\
& +\left(q^{3}+q^{5}+q^{7}+q^{9}\right) x_{11}+\left(1+q^{2}\right) x_{9}+\left(2 q^{2}+q^{4}\right) x_{8}+\left(q^{4}+2 q^{6}+q^{8}\right) x_{7} \\
& +q^{4} x_{5}+q^{6} x_{4}+\left(q^{4}+q^{6}\right) x_{3}+\left(q^{5}+q^{7}+q^{9}\right) x_{2}+q^{7} x_{1} .
\end{aligned}
$$

The coefficient of $x_{16}$ is not in $q \mathbb{Z}[q]$. Following the algorithm, we see that $G_{\lambda}\left(b_{\pi_{4}}\right)=$ $F_{\pi_{4}} v_{\lambda}-\left(q+q^{-1}\right) G_{\lambda}\left(b_{\pi_{1}}\right)$; we obtain

$G_{\lambda}\left(b_{\pi_{4}}\right)=x_{13}+q^{3} x_{12}+\left(q^{3}+q^{5}\right) x_{11}+q^{2} x_{8}+\left(q^{4}+q^{6}\right) x_{7}+q^{4} x_{5}+q^{6} x_{4}+q^{5} x_{2}+q^{7} x_{1}$.

Finally,

$$
\begin{aligned}
F_{\pi_{5}} v_{\lambda}= & x_{18}+\left(2 q+q^{-1}\right) x_{17}+\left(2 q^{3}+2 q+q^{-1}\right) x_{16}+\left(q+q^{3}\right) x_{15}+\left(2 q+q^{3}\right) x_{14} \\
& +\left(2 q^{4}+3 q^{2}+1\right) x_{13}+\left(q^{3}+q^{5}+q^{7}\right) x_{12}+\left(q+2 q^{3}+3 q^{5}+2 q^{7}+q^{9}\right) x_{11} \\
& +q^{3} x_{10}+\left(1+2 q^{2}+2 q^{4}\right) x_{9}+\left(2 q^{2}+3 q^{4}+q^{6}\right) x_{8} \\
& +\left(2 q^{4}+3 q^{6}+3 q^{8}+q^{10}\right) x_{7}+\left(q^{4}+q^{6}\right) x_{5}+q^{6} x_{4} \\
& +\left(q^{4}+q^{6}\right) x_{3}+\left(q^{5}+q^{7}+q^{9}\right) x_{2}+q^{7} x_{1} .
\end{aligned}
$$

We see that the highest basis vector not having a coefficient in $q \mathbb{Z}[q]$ (apart from $x_{18}$ ) is $x_{17}$. So we look at

$$
\begin{aligned}
F_{\pi_{5}} v_{\lambda}-\left(q+q^{-1}\right) G_{\lambda}\left(b_{\pi_{3}}\right)= & x_{18}+\left(q^{3}+q+q^{-1}\right) x_{16} \\
& +\left(q^{4}+2 q^{2}+1\right) x_{13}+\left(q^{4}+q^{2}+1\right)+\ldots
\end{aligned}
$$

(here, all the coefficients that have not been written lie in $q \mathbb{Z}[q]$ ). Now $x_{16}$ does not have a coefficient in $q \mathbb{Z}[q]$, so we look at

$$
F_{\pi_{5}} v_{\lambda}-\left(q+q^{-1}\right) G_{\lambda}\left(b_{\pi_{3}}\right)-\left(q+q^{-1}\right) G_{\lambda}\left(b_{\pi_{1}}\right)=x_{18}+\left(q^{2}+1\right) x_{13}+\ldots
$$

We see that

$$
G_{\lambda}\left(b_{\pi_{5}}\right)=F_{\pi_{5}} v_{\lambda}-\left(q+q^{-1}\right) G_{\lambda}\left(b_{\pi_{3}}\right)-\left(q+q^{-1}\right) G_{\lambda}\left(b_{\pi_{1}}\right)-G_{\lambda}\left(b_{\pi_{4}}\right) .
$$

REMARK. Let $\pi \in \Pi_{\lambda}$, and let $\phi(\pi)=s_{i_{1}} \ldots s_{i_{r}}$ be the reduced expression that is the smallest in the lexicographical order. Let

$$
F_{\pi}=F_{\alpha_{i_{1}}}^{\left(n_{1}\right)} \ldots F_{\alpha_{i_{r}}}^{\left(n_{r}\right)} .
$$

Write $\alpha=\alpha_{i_{1}}$. If $n_{1}>1$, then by [13, Lemma 5.3(b)], $\phi\left(e_{\alpha} \pi\right)=\phi(\pi)$, and hence

$$
F_{e_{\alpha} \pi}=F_{\alpha_{i_{1}}}^{\left(n_{1}-1\right)} \ldots F_{\alpha_{i_{r}}}^{\left(n_{r}\right)} \text {. }
$$

On the other hand, if $n_{1}=1$, then by [13, Lemma 5.3(a)] we see that $s_{\alpha} \phi\left(e_{\alpha} \pi\right) \nless{ }_{B} \phi\left(e_{\alpha} \pi\right)$. So by $\left[13\right.$, Lemma 5.3(b)], $\phi(\pi)=s_{\alpha} \phi\left(e_{\alpha} \pi\right)$. Therefore $\phi\left(e_{\alpha} \pi\right)=s_{i_{2}} \ldots s_{i_{r}}$, which is 
the smallest reduced expression (in the lexicographical order) for $\phi\left(e_{\alpha} \pi\right)$. Hence

$$
F_{e_{\alpha} \pi}=F_{\alpha_{i_{2}}}^{\left(n_{2}\right)} \ldots F_{\alpha_{i_{r}}}^{\left(n_{r}\right)} \text {. }
$$

The conclusion is that

$$
F_{\pi} \cdot v_{\lambda}=\frac{1}{\left[n_{1}\right]_{\alpha}} F_{\alpha} \cdot\left(F_{e_{\alpha} \pi} \cdot v_{\lambda}\right)
$$

So, in order to compute $F_{\pi} \cdot v_{\lambda}$, we have only to act with $F_{\alpha}$ on a vector that we have already computed.

REMARK. Instead of the algorithm described here for getting the monomials $F_{\pi}$, one can also follow the procedure outlined in [15] for constructing so-called 'adapted strings'. Instead of $\phi(\pi)$, this procedure uses the longest element in the Weyl group. However, the monomials that one finds in that case are generally different from the ones that we obtain. Moreover, they do not in general have the nice property described in the previous remark.

\section{The $A_{n}$-case}

In this section we assume that the root system $\Phi$ is of type $A_{n}$. We use results from [9] to show that in this case our algorithm is very much like the algorithm described in [11]. The simple roots are $\alpha_{1}, \ldots, \alpha_{n}$, where we use the usual ordering of the nodes of the Dynkin diagram (see [1]).

Since the fundamental weights are all minuscule, the corresponding irreducible $U_{q^{-}}$ modules are easy to construct (see [6, Chapter 5A]). For $V\left(\lambda_{k}\right)$, we consider the set of sequences $S=\left\{\left(i_{1}, \ldots, i_{k}\right) \mid 1 \leqslant i_{1}<i_{2}<\ldots<i_{k} \leqslant n+1\right\}$. Let $V$ be the vectorspace over $\mathbb{Q}(q)$ spanned by $v_{s}$ for $s \in S$. Let $s \in S$. If $i$ occurs in $s$, but $i+1$ does not, then we let $s^{i-}$ be the sequence obtained from $s$ by replacing $i$ by $i+1$, and we set $v_{s}^{i-}=v_{s^{i-}}$. Otherwise, $v_{s}^{i-}=0$. Also, if $i+1$ occurs in $s$, but $i$ does not, then we let $s^{i+}$ be the sequence obtained from $s$ by replacing $i+1$ by $i$, and we set $v_{s}^{i+}=v_{s^{i+}}$. Otherwise, $v_{s}^{i+}=0$. Now a $U_{q}$-action on $V$ is defined by $F_{\alpha_{i}} \cdot v_{s}=v_{s}^{i-}, E_{\alpha} \cdot v_{s}=v_{s}^{i+}$, and

$$
K_{\alpha_{i}} \cdot v_{s}= \begin{cases}q v_{s}, & \text { if } i \in S \text { and } i+1 \notin S, \\ q^{-1} v_{s}, & \text { if } i \notin S \text { and } i+1 \in S, \\ v_{s}, & \text { otherwise. }\end{cases}
$$

Then the $U_{q}$-module $V$ is isomorphic to $V\left(\lambda_{k}\right)$. To see this, we note that $v_{s}$ is a weight vector of weight $\mu_{s}=a_{1} \lambda_{1}+\ldots+a_{n} \lambda_{n}$, where $a_{i}=1$ if $i \in S, i+1 \notin S$; $a_{i}=-1$ if $i \notin S, i+1 \in S$; and $a_{i}=0$ otherwise. Set $s_{\lambda_{k}}=(1,2, \ldots, k)$; then $\mu_{s_{\lambda_{k}}}=\lambda_{k}$. If $i \in S$ and $i+1 \notin S$, then $s_{\alpha_{i}}\left(\mu_{S}\right)=\mu_{s i-}$. Since all elements of $S$ can be obtained from $s_{\lambda_{k}}$ by a sequence of 'moves' $s \rightarrow s^{i-}$ (see [9, Proposition 3.3.1]), we find that $\left\{\mu_{s} \mid s \in S\right\}=W \cdot \lambda_{k}$. Finally, we compare this with [6, 5A.1].

Let $\mathcal{L}\left(\lambda_{k}\right)$ be the $A$-submodule of $V$ spanned by the $v_{s}$, and let $\mathscr{B}\left(\lambda_{k}\right)$ be the set of all $v_{s} \bmod q \mathcal{L}\left(\lambda_{k}\right)$. Then $\left(\mathcal{L}\left(\lambda_{k}\right), \mathscr{B}\left(\lambda_{k}\right)\right)$ is a crystal base of $V$ (see [6, Lemma 9.6]). Furthermore, $C_{k}=\left\{v_{s} \mid s \in S\right\}$ is the canonical basis of $V$. (Indeed, the $v_{s}$ are certainly invariant under - because they are of the form $F_{\alpha_{i_{1}}} \ldots F_{\alpha_{i_{r}}} \cdot v_{s_{\lambda_{k}}}$. Secondly, $\left\{v_{s} \bmod q \mathcal{L}\left(\lambda_{k}\right) \mid s \in S\right\}=\mathscr{B}\left(\lambda_{k}\right)$.)

So the elements of $\mathcal{B}\left(\lambda_{k}\right)$ are labeled by the elements of $S$. From [9] we obtain the action of the Kashiwara operators as follows: $\widetilde{F}_{\alpha_{i}}\left(v_{s}\right)=v_{s}^{i-} \bmod q \mathcal{L}\left(\lambda_{k}\right)$, and $\widetilde{E}_{\alpha_{i}}\left(v_{s}\right)=$ $v_{s}^{i+} \bmod q \mathcal{L}\left(\lambda_{k}\right)$. 
We write a sequence $s=\left(i_{1}, \ldots, i_{k}\right)$ as a diagram with one column of length $k$ and the elements $i_{1}, \ldots, i_{k}$ from top to bottom. For example, the sequence $(1,4,5)$ is

$$
\begin{array}{|l|}
\hline 1 \\
\hline 4 \\
\hline 5 \\
\hline
\end{array}
$$

Now let $\lambda=a_{1} \lambda_{1}+\ldots+a_{n} \lambda_{n}$ be a dominant weight, and consider the tensor product $W=V\left(\lambda_{1}\right)^{\otimes a_{1}} \otimes \ldots \otimes V\left(\lambda_{n}\right)^{\otimes a_{n}}$. The basis elements are labelled by tableaux with $a_{n}+a_{n-1}+\ldots+a_{1}$ columns. The first $a_{n}$ columns have length $n$, the following $a_{n-1}$ columns have length $n-1$, and so on. The tableaux are filled with elements of $\{1,2, \ldots, n+1\}$, such that every column is strictly increasing. Then every column of length $k$ determines a basis element of $V\left(\lambda_{k}\right)$. Tensored together, they form a basis element of $W$; for example,

$$
\begin{array}{|l|l|l|}
\hline 1 & 1 & 4 \\
\hline 2 & 3 & =4 \\
\hline 3 & \multicolumn{2}{|c}{\otimes}
\end{array} \otimes \begin{array}{|l|}
\hline 1 \\
3
\end{array} \otimes \begin{array}{|l|}
\hline 1 \\
\hline 2 \\
\hline 3 \\
\hline
\end{array}
$$

Then the highest-weight vector $v_{\lambda}$, of weight $\lambda$ in $W$, is labelled by the tableau $T_{\lambda}$, where the $i$ th row contains only the number $i$. Let $V(\lambda)$ denote the submodule of $W$ generated by $v_{\lambda}$. Let $(\mathcal{L}(\lambda), \mathscr{B}(\lambda))$ be the crystal base of $V(\lambda)$. Then, by [9], the elements of $\mathscr{B}(\lambda)$ are labelled by tableaux with non-decreasing rows. In particular, these tableaux label the points in the crystal graph. From [9], we obtain the following algorithm for computing $\widetilde{F}_{\alpha_{i}}(T)$ and $\widetilde{E}_{\alpha_{i}}(T)$, where $T$ is such a tableau.

1. Write the numbers in the tableau as a sequence, starting from the top right, and going along the columns from right to left, top to bottom. Below each number, write a ' + ' if it is equal to $i$, a ' - ' if it is $i+1$, and a blank otherwise.

2. If there is a + followed by a - (maybe separated by blanks), then replace them by blanks. Continue until this operation is no longer possible.

3. (a) If there is no + left, then $\widetilde{F}_{\alpha_{i}}(T)=0$. Otherwise, change the $i$ corresponding to the leftmost + to an $i+1$. Rebuild the tableau, and the result is $\widetilde{F}_{\alpha_{i}}(T)$.

(b) If there is no - left, then $\widetilde{E}_{\alpha_{i}}(T)=0$. Otherwise, change the $i+1$ corresponding to the rightmost - into an $i$. Rebuild the tableau; the result is $\widetilde{E}_{\alpha_{i}}(T)$.

EXAMPLE 9. Let the root system be of type $A_{3}$, and set

$$
T=\begin{array}{|l|l|l|}
\hline 1 & 1 & 3 \\
\hline 2 & 2 & \\
\hline 3 & &
\end{array} .
$$

Then the sequence that we obtain is $3,1,2,1,2,3$. If $i=2$, this corresponds to $-o+o+-$ (where we represent a blank by ' $o$ '). After the operation of step 2, this becomes $-o+o o o$. We see that

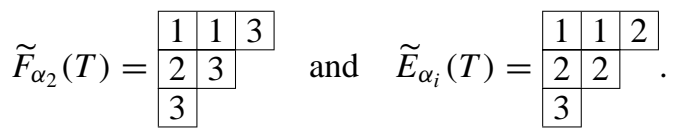

Now, from a tableau $T$ we obtain a monomial $F_{T}=F_{\alpha_{i_{1}}}^{\left(m_{1}\right)} \ldots F_{\alpha_{i p}}^{\left(m_{p}\right)}$ in the following way. Let $\pi$ be the path corresponding to $T$ (since the paths in $\Pi_{\lambda}$ label the points of the crystal graph, and so do the tableaux, there is a natural correpondence between the two). 
Let $\phi(\pi)=s_{i_{1}} \ldots s_{i_{p}}$ be the lexicographically smallest reduced expression for $\phi(\pi)$. We obtain the exponents $m_{1}, \ldots, m_{p}$ as follows. We let $m_{1}$ be maximal, such that

$$
\widetilde{E}_{\alpha_{i_{1}}}^{m_{1}}(T) \neq 0
$$

then $m_{2}$ is maximal, such that

$$
\widetilde{E}_{\alpha_{i_{2}}}^{m_{2}} \widetilde{E}_{\alpha_{i_{1}}}^{m_{1}}(T) \neq 0,
$$

and so on. Once we have the monomials $F_{T}$ for all the tableaux $T$, we proceed with the algorithm of Section 4.

The algorithm described in [11] for computing the canonical basis of $V(\lambda)$ has the same steps as our algorithm. First, for every tableau $T$, a monomial

$$
F_{T}=F_{\alpha_{i_{1}}}^{\left(n_{1}\right)} \ldots F_{\alpha_{i_{t}}}^{\left(r_{t}\right)}
$$

is computed. Secondly, from the vectors $F_{T} \cdot v_{\lambda}$ the canonical basis is computed using a triangular algorithm similar to the one that we use. Therefore, the main difference between the algorithms lies in the first step. We investigate this step a little further.

In $[11,4.1]$, the authors describe the following algorithm for obtaining a monomial $F_{T}$ from a tableau $T$. Let $i_{1}$ be the smallest index such that $i_{1}+1$ occurs in an $m$ th row of $T$, with $m \leqslant i_{1}$. Furthermore, $r_{1}$ is the number of occurrences of $i_{1}+1$ on an $m$ th row with $m \leqslant i$. Then $T_{1}$ is obtained from $T$ by replacing these $r_{1}$ occurrences of $i_{1}+1$ by $i_{1}$. Continuing with $T_{1}$ instead of $T$, we eventually arrive at the tableau $T_{\lambda}$, at which point the algorithm stops. We have obtained the sequences $i_{1}, \ldots, i_{t}$ and $r_{1}, \ldots, r_{t}$, and the monomial is

$$
F_{T}=F_{\alpha_{i_{1}}}^{\left(n_{1}\right)} \ldots F_{\alpha_{i_{t}}}^{\left(r_{t}\right)} .
$$

We note that applying $\widetilde{E}_{\alpha_{i}}$ amounts to replacing an $i+1$ by $i$. Since this $i+1$ was put there by a series of applications of $\widetilde{F}_{\alpha_{i_{k}}}$, starting with $T_{\lambda}$, we see that this $i+1$ must occur on the $m$ th row with $m \leqslant i$. By induction on the number of columns of $T$, it can be shown that if $i_{1}$ is minimal such that $i_{1}+1$ occurs in an $m$ th row of $T$ with $m \leqslant i_{1}$, then $\widetilde{E}_{\alpha_{i_{1}}}(T) \neq 0$. So our algorithm for obtaining the monomials $F_{T}$ is quite similar to that of [11]. However, in our algorithm we follow the lexicographically smallest reduced expression of a word in the Weyl group, in order to find the sequence $i_{1}, \ldots$ This means that sometimes we obtain a different monomial from that found with the algorithm from [11], as the following example shows.

EXAMPLE I0. Set

$$
T=\begin{array}{|l|l|l|}
\hline 1 & 1 & 4 \\
\hline 2 & 3 & \multicolumn{1}{|r}{} \\
\cline { 1 - 1 } 3 & \multicolumn{2}{|l}{}
\end{array} .
$$

Then the monomial obtained by the algorithm of [11] is $F_{\alpha_{2}} F_{\alpha_{3}} F_{\alpha_{2}} F_{\alpha_{1}}$. Let $\pi$ be the corresponding path; then $\phi(\pi)=s_{\alpha_{3}} s_{\alpha_{2}} s_{\alpha_{1}}$. This means that the monomial that we obtain is $F_{\alpha_{3}} F_{\alpha_{2}}^{(2)} F_{\alpha_{1}}$.

We conclude that in the $A_{n}$-case our algorithm is very similar to, but not the same as, the algorithm described in [11].

\section{Practical experiences}

In this section we discuss some practical experiences with an implementation of the algorithm inside the package QuaGroup [3], which was written in the computer algebra system GAP4 [2]. 
Let $\lambda=n_{1} \lambda_{1}+\ldots+n_{l} \lambda_{l}$ be a dominant weight. The input to the algorithm is $\lambda$, along with the modules $V\left(\lambda_{i}\right)$, for $1 \leqslant i \leqslant l$. These modules are given by their canonical bases. Furthermore, for a generator $F_{\alpha}$ and an element $v$ of the canonical basis of a $V\left(\lambda_{i}\right)$, we can compute $F_{\alpha} \cdot v$ by a table lookup.

We recall the notation used in Section 4: $\lambda=\mu_{1}+\ldots+\mu_{r}$, where $\mu_{i}=\lambda_{j_{i}}$. We work inside the tensor product $W(\lambda)=V\left(\mu_{1}\right) \otimes \ldots \otimes V\left(\mu_{r}\right)$, with basis $C=C_{1} \otimes \ldots \otimes C_{r}$. We set $v_{\lambda}=v_{\mu_{1}} \otimes \ldots \otimes v_{\mu_{r}}$, where $v_{\mu_{i}}$ is a highest-weight vector of $V\left(\mu_{i}\right)$. Furthermore, the length of an element of $w \in W(\lambda)$ is the number of elements of $C$ that occur in the expression of $w$ as a linear combination of elements of $C$.

The algorithm consists of two steps. In the first step, we express $F_{\pi} \cdot v_{\lambda}$ as a linear combination of elements of $C$. As explained at the end of Section $4, F_{\pi} \cdot v_{\lambda}=\zeta F_{\alpha} \cdot\left(F_{\pi^{\prime}} \cdot v_{\lambda}\right)$, where $\zeta \in \mathbb{Q}(q)$ and $F_{\pi^{\prime}} \cdot v_{\lambda}$ is a vector that we also have to compute. It follows that for each $F_{\pi} \cdot v_{\lambda}$ we have to compute one image $F_{\alpha} \cdot v$. Hence in the first step we compute $\operatorname{dim} V(\lambda)$ times an image $F_{\alpha} \cdot v$. In the second step we add some elements of the canonical basis (which we have already computed) to the element $F_{\pi} \cdot v_{\lambda}$. The number of these additions for each $F_{\pi} \cdot v_{\lambda}$ is clearly bounded by $\operatorname{dim} V(\lambda)$. Hence the total number of additions is bounded by $(\operatorname{dim} V(\lambda))^{2}$.

So the total number of operations (computing an image $F_{\alpha} \cdot v$, adding one element of $W(\lambda)$ to another) is bounded by a polynomial in $\operatorname{dim} V(\lambda)$. The cost of these operations is polynomial in the length of the elements that occur. Now the dimension of $W(\lambda)$ is exponential in the $n_{i}$. Therefore, an element of $W(\lambda)$ may have a length that is exponential in the $n_{i}$. So the question is whether elements of that kind occur during the execution of the algorithm.

Table 1 contains experimental data obtained using the algorithm. The computations were done on a Linux system with a $600 \mathrm{MHz}$ Pentium III processor and 64MB of working memory for GAP. We see that the algorithm is efficient enough to be able to compute canonical bases of modules of dimensions into the thousands. We also see that a jump in the maximal length of the elements that occur in the algorithm corresponds to a jump in the running time of the algorithm. This confirms the conclusion of the arguments given above, namely that the length of the elements that occur is an important factor in the running time.

The data given here do not lead to a conclusion as to whether the algorithm has a polynomial- or an exponential-time complexity. However, they do indicate that the maximal length of an element - and the running time - can increase rather rapidly if $\operatorname{dim} V(\lambda)$ increases. This is seen most spectacularly in the case of $G_{2}$.

\section{References}

1. N. Bourbaki, Groupes et algèbres de Lie (Hermann, Paris, 1968) Chapters 4, 5 and 6. 113,117

2. The GAP GrouP, 'GAP - Groups, algorithms, and programming, Version 4.3', 2002; http://www.gap-system.org. 111,115

3. W. A. DE GRAAF, 'QuaGroup, a GAP share package', 2001; http://www. math.uu.nl/people/graaf/quagroup.html. 111, 115

4. W. A. DE GraAF, 'Constructing canonical bases of quantized enveloping algebras', Experiment. Math. 11 (2002) 161-170. 105, 105

5. W. A. DE GRAAF, 'Five constructions of representations of quantum groups', preprint, 2002. 105 
Table 1: Running times for the computation of the canonical basis. The second column contains the highest weight, where we abbreviate $n_{1} \lambda_{1}+\ldots+n_{l} \lambda_{l}$ by $\left(n_{1}, \ldots, n_{l}\right)$. The same ordering of the fundamental weights as in [1] is used. The third column contains the dimension of the corresponding module. The fourth column displays the running time in seconds. The last column contains the maximal length of an element of the canonical basis.

\begin{tabular}{ccrrr}
\hline type & weight $\lambda$ & $\operatorname{dim} V(\lambda)$ & time $(\mathrm{s})$ & max. length \\
\hline$B_{4}$ & $(1,0,1,0)$ & 594 & 27 & 6 \\
$B_{4}$ & $(0,1,1,0)$ & 1650 & 146 & 18 \\
$B_{4}$ & $(1,1,0,1)$ & 2560 & 495 & 29 \\
$B_{4}$ & $(1,0,1,1)$ & 5040 & 1571 & 78 \\
$C_{3}$ & $(2,0,1)$ & 216 & 26 & 16 \\
$C_{3}$ & $(1,1,1)$ & 512 & 95 & 32 \\
$C_{3}$ & $(0,2,1)$ & 616 & 222 & 63 \\
$C_{3}$ & $(2,0,2)$ & 1078 & 1013 & 158 \\
$G_{2}$ & $(1,1)$ & 64 & 3 & 5 \\
$G_{2}$ & $(2,1)$ & 189 & 58 & 38 \\
$G_{2}$ & $(1,2)$ & 286 & 125 & 53 \\
$G_{2}$ & $(2,2)$ & 729 & 2610 & 439 \\
\hline
\end{tabular}

6. J. C. JAntzen, Lectures on quantum groups, Grad. Stud. in Math. 6 (Amer. Math. Soc., Providence, RI, 1996). 105, 106, 106, 106, 106, 107, 107, 107, 107, 107, 108, 109, 109, 111, 113, 113, 113

7. S. KANG and K. C. MisRA, 'Crystal bases and tensor product decompositions of $U_{q}\left(G_{2}\right)$-modules', J. Algebra 163 (1994) 675-691. 111

8. M. KASHIWARA, 'Similarity of crystal bases', Lie algebras and their representations (Seoul, 1995) (ed. S. Kang, M. Kim and I. Lee, Amer. Math. Soc., Providence, RI, 1996) 177-186. 107, 110

9. M. Kashiwara and T. NAKashima, 'Crystal graphs for representations of the $q$ analogue of classical Lie algebras', J. Algebra 165 (1994) 295-345. 113, 113, 113, 114,114

10. V. LaKshmibai, 'Bases for quantum Demazure modules', Representations of groups (Banff, AB, 1994) (ed. B. Allison and G. Cliff, Amer. Math. Soc., Providence, RI, 1995) 199-216. 105, 110, 110

11. B. LECLERC and P. TOFFIN, 'A simple algorithm for computing the global crystal basis of an irreducible $U_{q}\left(\mathrm{sl}_{n}\right)$-module', Internat. J. Algebra Comput. 10 (2000) 191-208. $105,105,105,113,115,115,115,115,115,115$

12. C. Lecouvey, 'An algorithm for computing the global basis of an irreducible $U_{q}\left(\mathfrak{s p}_{2 n}\right)$-module', Adv. in Appl. Math. 29 (2002) 46-78. 105, 105 
13. P. Littelmann, 'A Littlewood-Richardson rule for symmetrizable Kac-Moody algebras', Invent. Math. 116 (1994) 329-346. 107, 110, 112, 112, 112

14. P. Littelmann, 'Paths and root operators in representation theory', Ann. of Math. (2) 142 (1995) 499-525. 107

15. P. Littelmann, 'Cones, crystals, and patterns', Transform. Groups 3 (1998) 145-179. 113

16. G. Lusztig, 'Canonical bases arising from quantized enveloping algebras II', Common trends in mathematics and quantum field theories (Kyoto, 1990), Progr. Theoret. Phys. Suppl. 102 (1991) 75-201. 107

17. G. Lusztig, Introduction to quantum groups (Birkhäuser Boston Inc., Boston, MA, 1993). 105, 107, 108, 108, 108, 108, 108, 108

Willem A. de Graaf degraafemath.uu.nl

http: //www.math.uu.nl/people/graaf/

Mathematical Institute

University of Utrecht

The Netherlands 